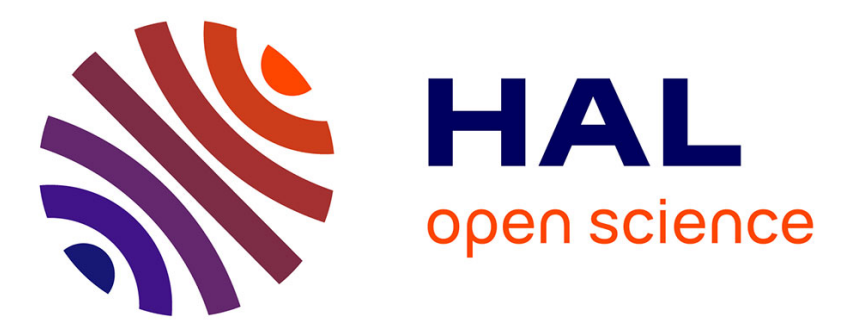

\title{
Spectre radiofréquence du formaldéhyde. Substitutions isotopiques
}

\author{
J.C. Chardon, C. Genty, D. Guichon, N. Sungur, J.G. Theobald
}

\section{To cite this version:}

J.C. Chardon, C. Genty, D. Guichon, N. Sungur, J.G. Theobald. Spectre radiofréquence du formaldéhyde. Substitutions isotopiques. Revue de Physique Appliquée, 1974, 9 (6), pp.961-965. 10.1051/rphysap:0197400906096100 . jpa-00243866

\section{HAL Id: jpa-00243866 https://hal.science/jpa-00243866}

Submitted on 1 Jan 1974

HAL is a multi-disciplinary open access archive for the deposit and dissemination of scientific research documents, whether they are published or not. The documents may come from teaching and research institutions in France or abroad, or from public or private research centers.
L'archive ouverte pluridisciplinaire HAL, est destinée au dépôt et à la diffusion de documents scientifiques de niveau recherche, publiés ou non, émanant des établissements d'enseignement et de recherche français ou étrangers, des laboratoires publics ou privés. 
Classification

Physics Abstracts

$5.445-5.449$

\title{
SPECTRE RADIOFRÉQUENCE DU FORMALDÉHYDE SUBSTITUTIONS ISOTOPIQUES
}

\author{
J. C. ChARDON, C. GENTY, D. GUICHON, N. SUNGUR et J. G. THEOBALD
}

Laboratoire de Spectroscopie Hertzienne et d'Electronique

Faculté des Sciences de Besançon, France

(Reçu le 4 juin 1974)

\begin{abstract}
Résumé. - A l'aide d'un spectromètre à jet moléculaire, nous avons étudié la structure hyperfine du spectre radiofréquence de dédoublement $\mathrm{K}$ de $\mathrm{H}_{2}{ }^{13} \mathrm{CO}$ et $\mathrm{D}_{2} \mathrm{CO}$. Chaque raie a une structure hyperfine qui nous permet de déterminer certaines des constantes moléculaires. On détermine les constantes d'interaction spin-rotation $M_{\mathrm{gg}}$ et la constante d'interaction quadripolaire $\chi_{\mathrm{aa}}$ du deutérium.

Abstract. - Using a molecular beam spectrometer, we study the hyperfine structure of the radiofrequency spectrum of $\mathrm{K}$ doubling in formaldehyde $\mathrm{H}_{2}{ }^{13} \mathrm{CO}$ and $\mathrm{D}_{2} \mathrm{CO}$. Each line has a hyperfine structure which permits us to determine certain molecular constants. We study the spin-rotation constants $M_{\mathrm{gg}}$ and the deuteron quadrupolar coupling constant $\chi_{\mathrm{aa}}$.
\end{abstract}

1. Introduction. - La molécule de formaldéhyde est une toupie légèrement asymétrique, les niveaux d'énergie sont des doublets $K$. Nous avons construit [1] un spectromètre à jet moléculaire avec lequel nous avons étudié les transitions dipolaires électriques de la branche $\mathrm{Q}$ de $\mathrm{H}_{2} \mathrm{CO}$ dans la région des radiofréquences. Nous avons observé la structure hyperfine des niveaux de rotation due au spin des deux protons. Ces effets hyperfins proviennent des interactions spin-rotation et spin-spin. Ces expériences ont été décrites dans une publication précédente $[2,3]$ et maintenant nous décrirons des expériences sur $\mathrm{H}_{2}{ }^{13} \mathrm{CO}$ (en abondance naturelle) et $\mathrm{D}_{2} \mathrm{CO}$. La structure hyperfine du spectre hyperfréquence (transition $1_{11}-1_{10}$ ) $a$ été étudiée précédemment par Flygare [4] $\left(\mathrm{D}_{2} \mathrm{CO}\right)$ et par Flygare et Weiss [5] $\left(\mathrm{H}_{2}{ }^{13} \mathrm{CO}\right)$. Nous donnerons d'abord quelques indications sur l'appareillage et ensuite nous décrirons nos expériences sur la structure hyperfine des transitions entre les composantes des doublets $\mathbf{K}$.

2. Appareillage. - Le spectromètre à jet moléculaire [6] (Fig. 1) a deux focaliseurs électrostatiques éliminant les molécules peuplant les niveaux d'énergie

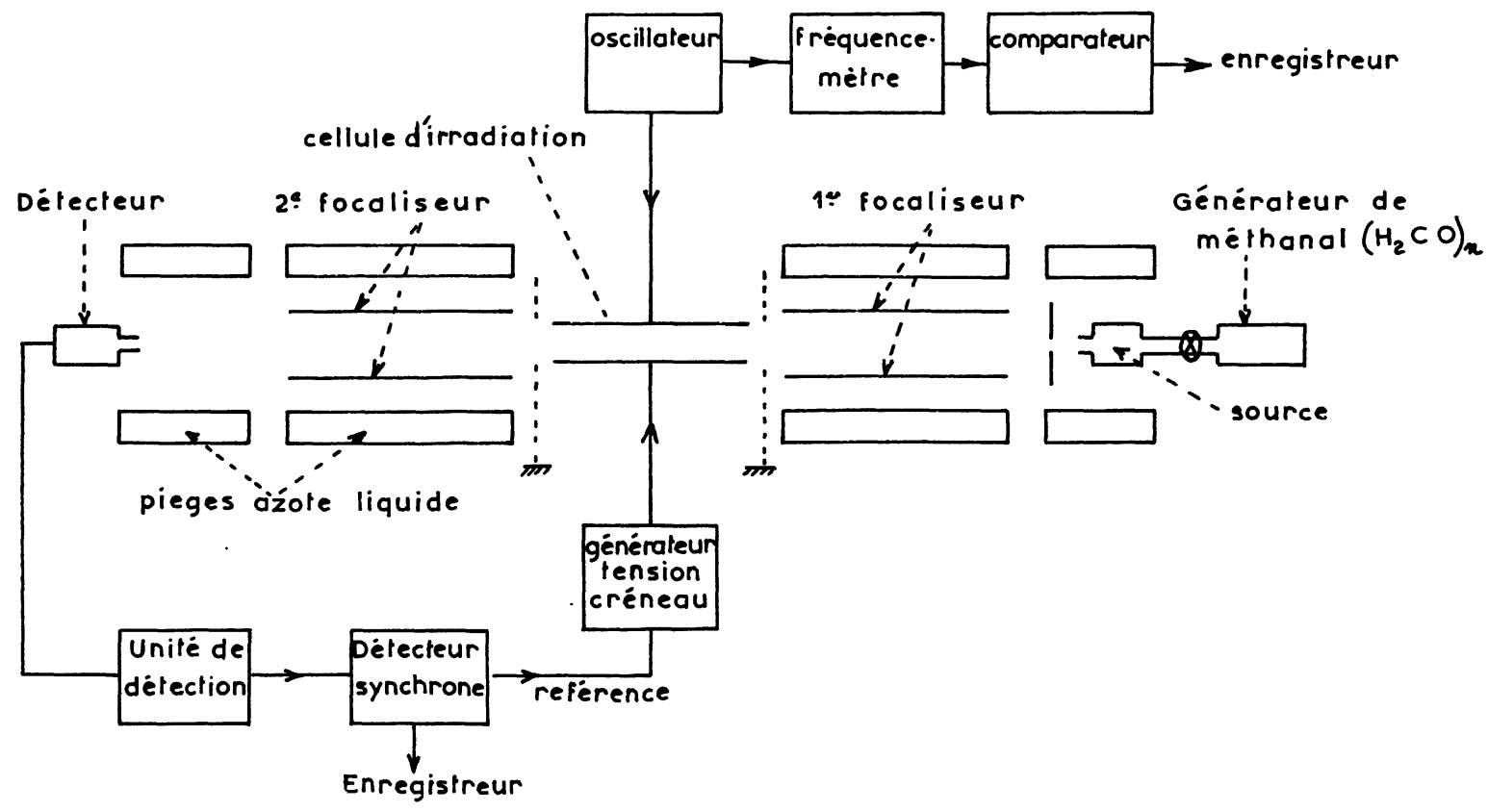

FIG. 1. - Schéma du spectromètre. 
inférieurs. Ces molécules sont piégées sur une enceinte refroidie à l'azote liquide. La cellule d'irradiation, placée entre les deux focaliseurs, est formée par les deux plateaux d'un condensateur. La détection du jet s'opère à l'aide d'une jauge à ionisation [6]. La pression dans le spectromètre est d'environ $10^{-6}$ torr. Le jet est obtenu par chauffage de trioxyméthylène. Le signal de radiofréquence est modulé (en créneaux) à une fréquence de $6 \mathrm{~Hz}$ en vue de la détection.

Nous balayons la fréquence d'irradiation et la résonance produit une diminution d'intensité du jet. La longueur de la cellule est $48 \mathrm{~cm}$ pour $\mathrm{D}_{2} \mathrm{CO}$, ce qui conduit à une largeur de raie de $1 \mathrm{kHz}$. Pour $\mathrm{H}_{2}{ }^{13} \mathrm{CO}$, la longueur est $27 \mathrm{~cm}$, parce que la sensibilité du spectromètre diminue avec la longueur du jet.

L'utilisation de formaldéhyde deutéré nous a conduits à étudier le rendement du spectromètre. Nous avons obtenu une consommation de $0,3 \mathrm{~g} / \mathrm{h}$ pour une pression source de 1 torr.

3. Récapitulation des formules donnant l'énergie hyperfine [7]. — L'hamiltonien s'écrit

$$
\mathscr{H}=\mathscr{H}_{\mathrm{R}}+\mathscr{H}_{\mathrm{SR}}+\mathscr{H}_{\mathrm{SS}}+\mathscr{H}_{\mathrm{Q}}
$$

$\mathcal{H}_{\mathrm{R}}$ est l'hamiltonien de rotation,

$\mathcal{H}_{\mathrm{SR}}$ est l'hamiltonien d'interaction spin-rotation $\mathcal{H}_{\mathrm{ss}}$ est l'hamiltonien d'interaction spin-spin $\mathscr{H}_{\mathrm{Q}}$ est l'hamiltonien d'interaction quadripolaire.

\subsection{L'HAMILTONIEN SPIN-ROTATION EST}

$$
\mathcal{H}_{\mathrm{SR}}=\sum_{\mathbf{g}} \frac{M_{\mathrm{gg}}<J_{\mathbf{g}}^{2}>}{J(J+1)} \mathbf{I} . \mathbf{J}
$$

$M_{\mathrm{gg}}$ est défini par Flygare [8].

a) Pour $\mathrm{H}_{2}{ }^{13} \mathrm{CO}$ le spectre est interprété comme provenant de l'interaction spin-rotation due à ${ }^{13} \mathrm{C}$, qui est supérieure aux interactions spin-spin et aux interactions spin-rotation des protons.

$$
\begin{aligned}
E_{\mathrm{SR}}=\frac{J(J+1)+I(I+1)-F(F+1)}{2 J(J+1)} & \\
& \times \sum_{\mathbf{g}} M_{\mathrm{gg}}<J_{\mathrm{g}}^{2}>.
\end{aligned}
$$

b) Pour $\mathrm{D}_{2} \mathrm{CO}$ ou $\mathrm{H}_{2} \mathrm{CO}$, on utilise le schéma de couplage $\mathbf{I}_{1}+\mathbf{I}_{2}=\mathbf{I}, \mathbf{I}+\mathbf{J}=\mathbf{F}$.

$$
\begin{aligned}
& E_{\mathrm{SR}}=\frac{J(J+1)+I(I+1)-F(F+1)}{4 J(J+1)} \times \\
& \times \sum_{\mathrm{K}=1,2} \sum_{\mathrm{g}} M_{\mathrm{Kgg}}<J_{\mathrm{g}}^{2}>
\end{aligned}
$$

l'indice $\mathrm{K}$ se rapportant au proton ou au noyau de deutérium.

\subsection{L'INTERACTION DIPOLAIRE SPIN-SPIN EST}

$$
\mathfrak{H}_{\mathrm{SS}}=\frac{\mu_{0}}{4 \pi} \beta^{2} g_{\mathrm{D}}^{2} r^{-3}\left[\mathbf{I}_{1} \cdot \mathbf{I}_{2}-3 r^{-2}\left(\mathbf{I}_{1} \cdot \mathbf{r}\right)\left(\mathbf{I}_{2} \cdot \mathbf{r}\right)\right] .
$$

Elle peut être écrite [9]

$$
\begin{aligned}
\mathscr{H}_{\mathrm{SS}}=-\frac{3 \mu_{0}}{4 \pi} \sqrt{5} & g_{\mathrm{D}}^{2} \beta^{2} r^{-5} \times \\
& \times\left\{\left\{r^{(1)} r^{(1)}\right\}^{(2)}\left\{I_{1}^{(1)} I_{2}^{(1)}\right\}^{(2)}\right\}^{(0)}
\end{aligned}
$$

en utilisant les tenseurs sphériques.

Les éléments de matrice correspondants sont

$$
\begin{gathered}
<\left(I_{\mathrm{D}} I_{\mathrm{D}}\right) I J \tau F M_{\mathrm{F}}\left|\mathcal{H}_{\mathrm{SS}}\right|\left(I_{\mathrm{D}} I_{\mathrm{D}}\right) I^{\prime} J \tau F M_{\mathrm{F}}>= \\
=-(-1)^{\mathrm{F}+J+I^{\prime}} \frac{3 \mu_{0}}{4 \pi} g_{\mathrm{D}}^{2} \beta^{2} r^{-5}\left\{\begin{array}{ccc}
F & I^{\prime} & J \\
2 & J & I
\end{array}\right\} \times \\
\times<I_{\mathrm{D}} I_{\mathrm{D}} I\left\|\left\{I_{\mathrm{D}}^{(1)} I_{\mathrm{D}}^{(1)}\right\}^{(2)}\right\| I_{\mathrm{D}} I_{\mathrm{D}} I^{\prime}> \\
\times<J \tau\left\|\left\{r^{(1)} r^{(1)}\right\}^{(2)}\right\| J \tau>.
\end{gathered}
$$

Le coefficient $6 j$ est nul sauf si $I^{\prime}-I=0, \pm 1, \pm 2$.

La fonction d'onde totale est symétrique dans l'opération de permutation des 2 noyaux de deutérium. Dans le cas de la transition $3_{30}-3_{31}$ étudiée, la fonction de rotation est antisymétrique car $\mathrm{K}_{-1}$ est impair. La fonction de spin doit alors être antisymétrique, donc $I=1$. Les seuls éléments de matrice non nuls correspondent à $I=I^{\prime}=1$. Les éléments de matrice réduits sont donnés par Bluyssen [10]. On a :

$$
\begin{aligned}
E_{\mathrm{SS}} & =-\frac{\mu_{0} g_{\mathrm{D}}^{2} \beta^{2}}{4 \pi r^{5}} \times \\
& \times \frac{I(I+1)+4 I_{\mathrm{D}}\left(I_{\mathrm{D}}+1\right)}{(2 I-1)(2 I+3) J(J+1)(2 J+3)(2 J-1)} \\
& \times \sum_{\mathrm{g}} r_{\mathrm{g}}^{2}\left[3<J_{\mathrm{g}}^{2}>-J(J+1)\right] \\
& \times\left[3\left(\frac{X}{2}\right)^{2}-\frac{3}{2}\left(\frac{X}{2}\right)-I(I+1) J(J+1)\right]
\end{aligned}
$$

où $X=J(J+1)+I(I+1)-F(F+1)$.

3.3 L'HAMILTONIEN D'INTERACTION QUADRIPOLAIRE ÉLECTRIQUE. Il peut s'écrire comme un produit scalaire $[11,12]$ :

$$
\mathscr{H}_{\mathrm{Q}}=\sum_{\mathrm{K}=1,2}\left(V^{(2)} \cdot Q^{(2)}\right)_{\mathrm{K}}
$$

de deux opérateurs tensoriels sphériques. $Q^{(2)}$ et $V^{(2)}$ sont les tenseurs moment quadripolaire et gradient de champ donnés par les expressions (III.39) et (III.40) de Ramsey [12]. Les éléments de matrice sont :

$$
\begin{aligned}
<\left(I_{\mathrm{D}} I_{\mathrm{D}}\right) & I J \tau F M_{\mathrm{F}}\left|\mathcal{H}_{\mathrm{Q}}\right|\left(I_{\mathrm{D}} I_{\mathrm{D}}\right) I^{\prime} J \tau F M_{\mathrm{F}}>= \\
= & 2(-1)^{J+F}\left[(2 I+1)\left(2 I^{\prime}+1\right)\right]^{1 / 2} \\
& \times\left\{\begin{array}{lll}
I_{\mathrm{D}} & I^{\prime} & I_{\mathrm{D}} \\
I & I_{\mathrm{D}} & 2
\end{array}\right\}\left\{\begin{array}{lll}
F & J & I \\
2 & I^{\prime} & J
\end{array}\right\} \\
& \times<I_{\mathrm{D}}\left\|Q^{(2)}\right\| I_{\mathrm{D}}><J \tau\left\|V^{(2)}\right\| J \tau>.
\end{aligned}
$$


Nous avons :

$$
\begin{aligned}
<I_{\mathrm{D}} \| Q^{(2)} & \| I_{\mathrm{D}}>= \\
= & \frac{e Q}{2}\left[\frac{\left(2 I_{\mathrm{D}}+3\right)\left(2 I_{\mathrm{D}}+2\right)\left(2 I_{\mathrm{D}}+1\right)}{2 I_{\mathrm{D}}\left(2 I_{\mathrm{D}}-1\right)}\right]^{1 / 2}
\end{aligned}
$$

et

$$
\begin{aligned}
<J \tau\left\|V^{(2)}\right\| J \tau>= & \\
& =\frac{q_{J}}{2}\left[\frac{(2 J+3)(2 J+2)(2 J+1)}{2 J(2 J-1)}\right]^{1 / 2} .
\end{aligned}
$$

Les éléments diagonaux s'écrivent :

$$
\begin{aligned}
& E_{\mathrm{Q}}=\frac{e q_{J} Q}{2 I_{\mathrm{D}}\left(2 I_{\mathrm{D}}-1\right) J(2 J-1)} \times \\
& \times\left[1-\frac{I(I+1)+4 I_{\mathrm{D}}\left(I_{\mathrm{D}}+1\right)}{(2 I-1)(2 I+3)}\right] \\
& \times\left[\frac{3 X}{4}(X-1)-I(I+1) J(J+1)\right]
\end{aligned}
$$

avec :

$$
\begin{aligned}
e q_{J} Q=\frac{\sum_{\mathbf{g}}\left(\chi_{1 \mathrm{gg}}+\chi_{2 \mathrm{gg}}\right)<J_{\mathrm{g}}^{2}>}{(J+1)(2 J+3)} & = \\
& =\frac{2 \alpha^{\mathrm{Q}}}{(J+1)(2 J+3)} .
\end{aligned}
$$

4. Résultats. - 4.1 ObSeRvation De Quelques TRANSITIONS DE $\mathrm{H}_{2}{ }^{13} \mathrm{CO}, \mathrm{H}_{2} \mathrm{C}^{18} \mathrm{O}$ eT $\mathrm{D}_{2} \mathrm{CO}$.

Nous avons d'abord observé les transitions basse fréquence de la branche $\mathrm{Q}$ de $\mathrm{H}_{2} \mathrm{CO}$ entre $100 \mathrm{kHz}$ et $300 \mathrm{MHz}$ [2]. La sensibilité de l'appareillage nous a ensuite permis d'observer quelques raies du spectre basse fréquence de $\mathrm{H}_{2}{ }^{13} \mathrm{CO}$ et $\mathrm{H}_{2} \mathrm{C}^{18} \mathrm{O}$ dans un jet de formaldéhyde obtenu par décomposition thermique du trioxyméthylène, les deux espèces isotopiques existant en abondance naturelle. D'autres expériences ont été réalisées sur $\mathrm{D}_{2} \mathrm{CO}$ obtenu par chauffage d'un produit fourni par Merck. Les résultats expérimentaux obtenus sont rassemblés dans le tableau I. Dans ce tableau, nous avons aussi reporté les valeurs des fréquences calculées à l'ordre $(K+2)$ par la méthode de Kivelson [13] en prenant les constantes de rotation [14] du tableau II ( $\beta$ est le paramètre d'asymétrie). Les corrections de distorsion centrifuge sont calculées par la méthode de Kivelson et Wilson [15] à partir des résultats de Oka et Morino [16]. Les écarts entre les valeurs expérimentales et les valeurs calculées sont dus en majeure partie à l'incertitude existant sur les valeurs des constantes moléculaires et les constantes de distorsion centrifuge. La comparaison des intensités des raies de $\mathrm{H}_{2} \mathrm{CO}$ et $\mathrm{H}_{2}{ }^{13} \mathrm{CO}$ permet une détermination du pourcentage de l'iso-

\section{TABLeau I}

Raies de dédoublement $\mathrm{K}$ de $\mathrm{H}_{2}{ }^{13} \mathrm{CO}, \mathrm{H}_{2} \mathrm{C}^{18} \mathrm{O}$ et $\mathrm{D}_{2} \mathrm{CO}$ dans la région radiofréquence

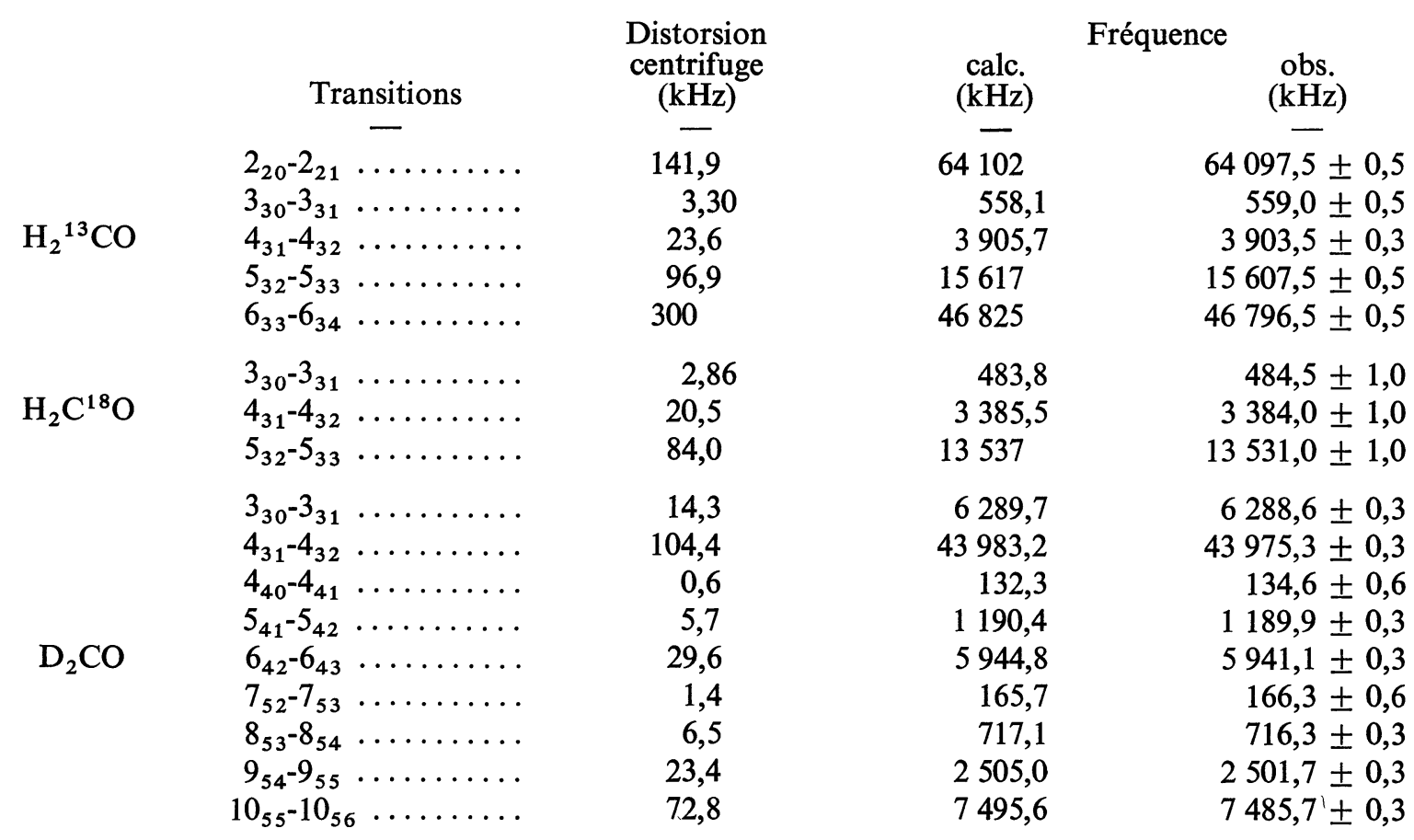

TABLEAU II

Valeurs des constantes de rotation

\begin{tabular}{cccccc} 
& \multicolumn{1}{c}{$\boldsymbol{A}$} & \multicolumn{1}{c}{$\boldsymbol{B}$} & \multicolumn{1}{c}{$\boldsymbol{C}$} & \multicolumn{1}{c}{$\boldsymbol{\beta}$} \\
$\mathrm{H}_{2}{ }^{13} \mathrm{CO}$ & $\ldots \ldots$ & 282038 & 37810,83 & 3321536 & $-0,009320$ \\
$\mathrm{H}_{2} \mathrm{C} 18 \mathrm{O}$ & $\ldots \ldots$ & 282029 & 36904,1 & 32513,0 & $-0,008877$ \\
$\mathrm{D}_{2} \mathrm{CO}$ & $\ldots \ldots$ & 141664 & 32283,11 & 26185,76 & $-0,027116$
\end{tabular}

tope ${ }^{13} \mathrm{C}$ dans le trioxyméthylène ; en tenant compte de l'efficacité des focaliseurs on trouve

$$
R=1,14 \pm 0,15 \%
$$

ce qui est en accord avec la valeur $1,108 \%$ de ${ }^{13} \mathrm{C}$ en abondance naturelle. 
4.2. INTERACTION SPIN-ROTATION DE ${ }^{13} \mathrm{C}$ DANS $\mathrm{H}_{2}{ }^{13} \mathrm{CO}$. - Le spin nucléaire de ${ }^{13} \mathrm{C}$ est $\frac{1}{2}$, les interactions hyperfines dans la molécule $\mathrm{H}_{2}{ }^{13} \mathrm{CO}$ sont d'origine magnétique. Puisque $F=J \pm \frac{1}{2}$ chaque état de rotation est décomposé en 2 niveaux hyperfins En champ d'irradiation faible, on n'observe qu'une raie qui correspond aux 2 transitions $\Delta F=0$ non résolues et dont les fréquences sont reportées dans le tableau I. La résolution de l'appareillage ne permet pas de résoudre ces deux transitions car leur écart est très petit; il est proportionnel à la différence des $\left\langle J_{\mathbf{g}}^{2}\right\rangle$ de l'état supérieur (s) et inférieur (i) des doublets $\mathrm{K}$ et puisque $\left\langle J_{\mathrm{g}}^{2}\right\rangle_{\mathrm{s}} \simeq\left\langle J_{\mathrm{g}}^{2}\right\rangle_{\mathrm{i}}$ nous avons calculé à partir des résultats de Bragg et Golden [19] $\frac{1}{2}\left[\left\langle J_{\mathrm{g}}^{2}\right\rangle_{\mathrm{s}}+\left\langle J_{\mathrm{g}}^{2}\right\rangle_{\mathrm{i}}\right]$ (tableau III). Les axes $\mathrm{g}=a$,

\section{Tableau III}

Valeurs de $<J_{\mathrm{g}}^{2}>$ pour $\mathrm{H}_{2}{ }^{13} \mathrm{CO}$

\begin{tabular}{|c|c|c|c|}
\hline Transitions & $<\underline{J_{c}^{2}}>$ & $<\underline{J_{b}^{2}}>$ & $<\underline{J_{a}^{2}}>$ \\
\hline $2_{20}-2_{21} \ldots \ldots$ & 0,987 & 1,013 & 3,999 \\
\hline $4_{31}-4_{32} \ldots \ldots$ & 5,43 & 5,57 & 8,999 \\
\hline $5_{32}-5_{33} \ldots \ldots$ & 10,34 & 10,66 & 8,9986 \\
\hline $6_{33}-6_{34} \ldots$ & 16,19 & 16,81 & 8,9973 \\
\hline
\end{tabular}

$b, c$ sont les axes principaux d'inertie, l'axe $a$ est dirigé suivant l'axe de symétrie de la molécule. Lorsque l'amplitude du champ d'irradiation croît, deux composantes hyperfines correspondant aux transitions $\Delta F= \pm 1$ apparaissent ; elles sont symétriques par rapport à la raie centrale qui s'élargit sous l'effet d'un début de saturation. La figure 2 représente les deux composantes hyperfines du doublet $2_{2}$. Dans le tableau IV nous avons reporté les valeurs des écarts hyperfins observés ainsi que l'intensité calculée des composantes hyperfines non saturées.

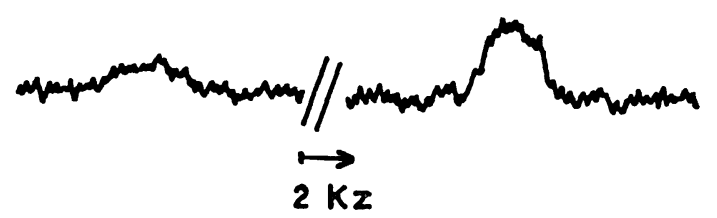

Fig. 2. - Structure hyperfine. Transition $\Delta F=1$ et $\Delta F=-1$ de la raie $2_{2}$ de $\mathrm{H}_{2}{ }^{13} \mathrm{CO}$.

TABLEAU IV

Ecarts hyperfins dus à l'interaction spin-rotation de ${ }^{13} \mathrm{C}$ dans $\mathrm{H}_{2}{ }^{13} \mathrm{CO}$

\begin{tabular}{|c|c|c|c|}
\hline \multirow[b]{2}{*}{ Transitions } & \multirow[b]{2}{*}{ Intensité } & \multicolumn{2}{|c|}{ Ecarts hyperfins } \\
\hline & & $\begin{array}{l}\text { Obs. } \\
(\mathrm{kHz})\end{array}$ & $\begin{array}{c}\text { Calc. } \\
\text { (kHz) }\end{array}$ \\
\hline $2_{20}-2_{21} \ldots \ldots$ & 4,00 & $224,6 \pm 0,3$ & 224,7 \\
\hline $4_{31}-4_{32} \ldots \ldots$ & 1,23 & $292,8 \pm 0,3$ & 292,9 \\
\hline $5_{32}-5_{33} \ldots \ldots$ & 0,826 & $263,7 \pm 0,3$ & 263,8 \\
\hline $6_{33}-6_{34} \ldots \ldots$ & 0,592 & $248,4 \pm 0,3$ & 248,4 \\
\hline
\end{tabular}

Par ailleurs, on constate que l'intensité maximale de la composante hyperfine de plus grande fréquence est toujours supérieure à celle de la raie inférieure. Sur la figure 2 on constate que le rapport est de l'ordre de 3. Cet effet s'explique en prenant en considération l'évolution des niveaux d'énergie d'une molécule de la sortie du premier focaliseur à l'entrée de la cellule d'irradiation. Sur la figure 3 , nous avons tracé le comportement qualitatif des niveaux d'énergie relatifs aux composantes supérieures du doublet $2_{2}$ d'une molécule au cours de son évolution dans cette région intermédiaire où le champ électrostatique varie. La variation du champ est assez lente pour que la plupart des molécules traversent cette région de manière adiabatique. A la sortie du focaliseur, les molécules focalisées sont dans un état $M_{J} \neq 0$. Donc, si la répartition des niveaux hyperfins est celle de la figure 3 (le niveau supérieur correspondant à $F=\frac{5}{2}$ ), les populations des niveaux $F=\frac{5}{2}$ et $F=\frac{3}{2}$ sont respectivement proportionnelles à 6 et 2 . A saturation on égalise les populations, et puisque toutes les molécules qui ont transité dans l'état inférieur du doublet $\mathrm{K}$ sont éliminées, on trouve que dans ce cas le rapport des intensités des deux raies observées est 3 . Par contre, si le niveau supérieur correspondait à $F=\frac{3}{2}$, une analyse analogue montre que les deux composantes hyperfines auraient la même intensité à saturation. Le niveau hyperfin de plus grande énergie correspond donc à $F=J+\frac{1}{2}$, et $\sum_{\mathbf{g}} M_{\mathrm{gg}}<J_{\mathrm{g}}^{2}>$ est négatif.

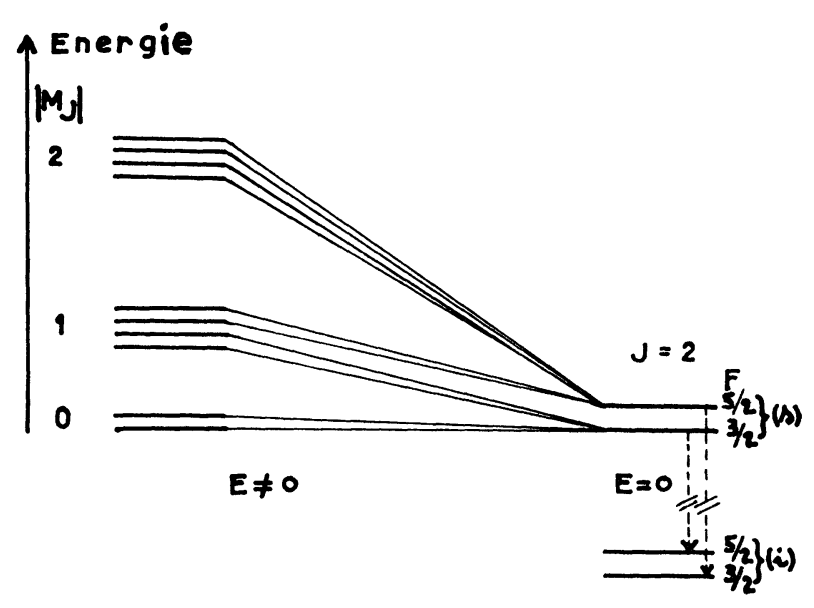

Fig. 3. - Evolution des niveaux d'énergie Stark lorsque le champ électrostatique varie $(J=2)$.

A partir des écarts hyperfins mesurés on obtient :

$$
\begin{aligned}
M_{\mathrm{aa}} & =-128,0 \pm 0,3 \mathrm{kHz} \\
M_{\mathrm{cc}}+M_{\mathrm{bb}} & =-27,1 \pm 0,5 \mathrm{kHz} .
\end{aligned}
$$

Puisque $M_{\text {aa }}$ est négatif, les effets électroniques sont supérieurs aux effets des charges nucléaires lors de la rotation autour de l'axe $a$. A partir de nos mesures, il est difficile de calculer séparément $M_{\mathrm{cc}}$ et $M_{\mathrm{bb}}$ avec précision; toutefois on trouve que la différence $M_{\mathrm{cc}}-M_{\mathrm{bb}}$ est positive; on peut l'estimer à 
TABLEAU V

Valeurs (en $\mathrm{kHz}$ ) des éléments diagonaux du tenseur d'interaction spin-rotation pour le proton, le deuton et ${ }^{13} \mathrm{C}$

Elément du tenseur spin-rotation

$$
\begin{aligned}
& \bar{M}_{\mathrm{aa}} \\
& M_{\mathrm{bb}} \\
& M_{\mathrm{cc}}
\end{aligned}
$$

Valeur mesurée pour le proton dans $\mathrm{H}_{2} \mathrm{CO}$

$$
\begin{aligned}
& 3,65 \pm 0,15 \\
& -2 \pm 0,15 \\
& 2,5 \pm 0,15
\end{aligned}
$$

Valeur calculée pour le deuton dans $\mathrm{D}_{2} \mathrm{CO}$

$$
\begin{array}{r}
0,28 \\
-0,25 \\
0,29
\end{array}
$$

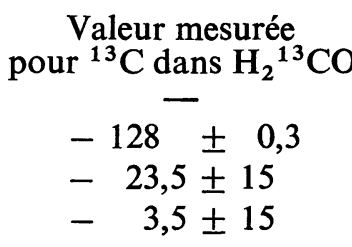

$20 \pm 15 \mathrm{kHz}$. Les valeurs des constantes obtenues sont reportées dans le tableau V. Dans la cinquième colonne du tableau IV nous avons reporté les valeurs des écarts hyperfins calculés à partir des constantes obtenues.

4.3 INTERACTION SPIN-ROTATION DES PROTONS DANS $\mathrm{H}_{2} \mathrm{CO}$. - Les expériences réalisées sur $\mathrm{H}_{2} \mathrm{CO}$ [3] avec une cellule d'irradiation de $48 \mathrm{~cm}$ nous ont permis d'observer la structure hyperfine sur une dizaine de transitions. Les valeurs obtenues des constantes de couplage spin-rotation du proton sont reportées dans le tableau V. En outre, on trouve $17,6 \pm 0,5 \mathrm{kHz}$ pour la constante d'interaction spin-spin, des protons, valeur qui est en accord avec celle calculée à partir de la structure moléculaire.

4.4 INTERACTION QUADRIPOLAIRE DU DEUTON DANS $\mathrm{D}_{2} \mathrm{CO}$. - Le spin du noyau de deutérium est 1 . La figure 4 représente le spectre de la transition $3_{30}-3_{31}$. Les états de rotation $3_{30}$ et $3_{31}$ sont décomposés en 3 niveaux hyperfins $F=J+1, J, J-1$. Sur le spectre on voit les composantes hyperfines relatives aux transitions $\Delta F= \pm 1$ symétriques par rapport à la raie centrale. Nous avons calculé les valeurs des constantes de couplage spin-rotation à partir des résultats obtenus sur $\mathrm{H}_{2} \mathrm{CO}$ (tableau V) ainsi que la valeur de la constante d'interaction spin-spin en

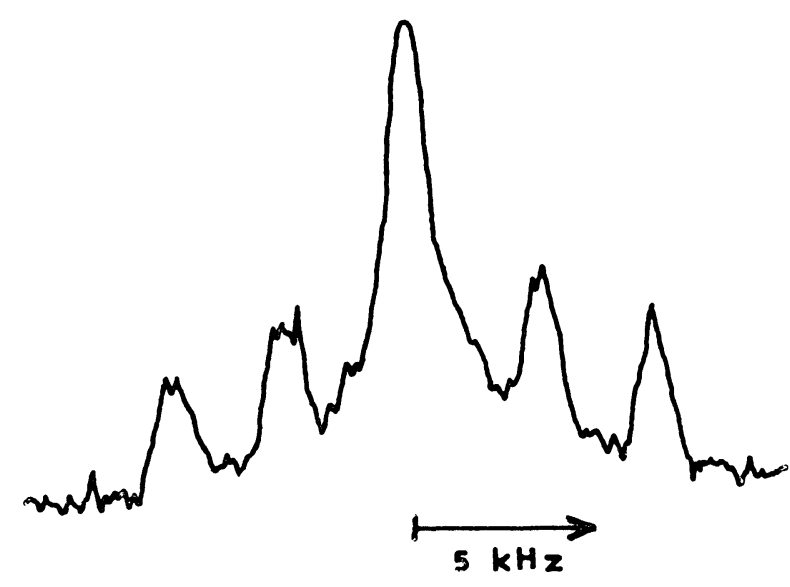

Fig. 4. - Structure hyperfine de la raie $3_{3}$ de $\mathrm{D}_{2} \mathrm{CO}$.

prenant [14] $r=1,892 \AA$. Le calcul de $\left\langle J_{\mathrm{g}}^{2}\right\rangle$ donne 9,$00 ; 1,55 ; 1,45$ respectivement pour $\mathrm{g}=a, b, c$. Connaissant ainsi les contributions magnétiques (spinrotation et spin-spin) à l'énergie hyperfine nous déterminons la contribution quadripolaire soit

$$
\alpha^{\mathrm{Q}}=-84,2 \mathrm{kHz} \text {. }
$$

En prenant [4] $\chi_{\mathrm{bb}}-\chi_{\mathrm{cc}}=183 \pm 8 \mathrm{kHz}$ on trouve $\chi_{\mathrm{aa}}=-11,8 \pm 0,5 \mathrm{kHz}$. Cette valeur est en accord avec celle trouvée par Flygare [4], toutefois, elle est plus précise que celle de cet auteur.

\section{Bibliographie}

[1] Genty, C., Gillet, D., Theobald, J. G., C. R. Hebd. Séan. Acad. Sci. B 268 (1969) 1499.

[2] Chardon, J. C. et Guichon, D., J. Physique 34 (1973) 791.

[3] Guichon, D., Thèse Besançon 1973.

[4] Flygare, W. H., J. Chem. Phys. 41 (1964) 206.

[5] Flygare, W. H., Weiss, V., J. Chem. Phys. 45 (1966) 2786.

[6] Genty, C., Thèse Besançon, 1970. GILlET, D., Thèse Besançon, 1970.

Genty, C., Theobald, J. G., Revue Phys. Appl. 8 (1973) 361.

[7] Thaddeus, P., Krisher, L. C., Loubser, L., J. Chem. Phys. 40 (1964) 257.

[8] Flygare, W. H., J. Chem. Phys. 41 (1964) 793.

[9] Judd, B. R., Operator Techniques in Atomic Spectroscopy (McGraw Hill Book Company, Inc. New York) 1963.

[10] Bluyssen, H., Thesis Nijmegen 1968. Huiszoon, C., Thesis Nijmegen 1966.
[11] Abragam, A., Principes du magnétisme nucléaire (P. U. F. Paris) 1961

[12] Ramsey, N. F., Molecular Beams (Oxford) 1956.

[13] Kivelson, D., J. Chem. Phys. 21 (1953) 536.

[14] Oka, T., Hirakawa, H., Shimoda, K., J. Phys. Soc. Japan 15 (1960) 2265.

TAKaGi, K., OKa, T., 18 (1963) 1174.

[15] Kivelson, D., Wilson, E. B., J. Chem. Phys. 20 (1952) 1575.

[16] OKa, T., Morino, Y., J. Phys. Soc. Japan 16 (1961) 1235.

[17] Chardon, J. C., Genty, C., Theobald, J. G., C. R. Hebd. Séan. Acad. Sci. 2788 (1974) 1.

[18] Chardon, J. C., Genty, C., Gillet, D., Mandret, G., Theobald, J. G., C. R. Hebd. Séan. Acad. Sci. 270 (1970) 1103.

[19] Bragg, J. K., Golden, S., Phys. Rev. 75 (1949) 735. 